\section{Gut punch to osteoporosis}

\author{
By Michael J. Haas, Senior Writer
}

U.S. and Indian researchers have shown that blocking the production of serotonin in the gut with a tryptophan hydroxylase 1 inhibitor from Lexicon Pharmaceuticals Inc. can increase bone formation to treat or prevent osteoporosis. ${ }^{1}$ The findings have prompted Lexicon to investigate whether its other TPH1 inhibitors, which are in Phase II testing for GI disorders, could be used to treat osteoporosis.

Tryptophan hydroxylase $1(\mathrm{TPH} 1 ; \mathrm{TPH})$ is an enzyme that plays a key role in the biosynthesis of gut-derived serotonin. In 2008, Lexicon published a paper in The Journal of Pharmacology and Experimental Therapeutics that described the discovery of small molecule THP1 inhibitors and their use to inhibit serotonin production in the gut to treat various GI conditions. ${ }^{2}$

That paper caught the eye of Columbia University's Gerard Karsenty and Patricia Ducy, who in the same year published that serotonin produced in the gut repressed bone formation in mice. ${ }^{3}$ With both sets of results in mind, the researchers theorized that blocking production of serotonin in the gut with a TPH1 inhibitor could prevent or treat osteoporosis by increasing bone formation.

A team co-led by Karsenty and Ducy found that Lexicon's small molecule TPH1 inhibitor LP533401 prevented and even reversed osteoporosis in mice by increasing bone mass, density and formation compared with no treatment. The inhibitor had no effect on brain levels of serotonin, indicating that it did not cross the blood brain barrier.

The biotech had not advanced the compound in favor of other TPH1 inhibitors. Donald Landry, chair of medicine at Columbia, told SciBX that Karsenty's team worked with Columbia's Organic Synthesis Collaborative Center to synthesize LP533401. Landry is executive director of the center.

In addition to Karsenty, chair of genetics and development at Columbia University Medical Center, and Ducy, assistant professor of pathology and cell biology at Columbia University, the team included researchers from the Indian Institute of Science, India's Central Drug Research Institute, Harvard Medical School and Dana-Farber Cancer Institute. Data were reported in Nature Medicine.

"The paper is very supportive of the hypothesis that by modulating peripheral serotonin you can induce bone formation" with an oral therapeutic, said Brian MacDonald, CEO of Zelos Therapeutics Inc. "If someone offered me the chance to work on this, I'd take it."

\section{Breaking it down}

MacDonald cautioned that the development of any new osteoporosis therapy would be measured against the efficacy of parathyroid hormone (PTH), which is the only drug approved for osteoporosis that promotes bone growth rather than stopping bone loss.

Eli Lilly and Co. markets Forteo teriparatide, an injectable recombinant PTH fragment, to treat osteoporosis. Nycomed markets Preotact recombinant PTH (NPSP558) in the EU to treat postmenopausal osteoporosis, and Nycomed's partner NPS Pharmaceuticals Inc. has the hormone in registration as Preos in the U.S. to treat postmenopausal osteoporosis.

"PTH is a good osteoporosis therapy that has had significant benefits in patients and is well accepted by prescribing specialists. While there is always room for improvement, it will be the agent to beat with new therapies" such as those that inhibit serotonin production, MacDonald said. "PTH typically reduces the incidence of fractures by $40 \%-70 \%$. A new therapy, whether as a standalone or in combination, could do better," but the hurdle is steep.

MacDonald did note that there is room for improvement in the anabolic (bone growing) space. "The bigger problems for uptake of PTH therapy have to do with the need for daily injections and restrictions on who can use it" due to a black box warning against treating patients with more severe disease, he added.

If a new therapy overcame that restriction, it would have the opportunity to expand into the severe disease population. "If you have a meaningful anabolic response with a non-PTH therapeutic, you could conceivably capture the whole market," said MacDonald.

Zelos' ZT-031, a cyclic 31-residue peptide analog of PTH, has completed Phase II testing to treat osteoporosis. The company's ZT-034, an intranasal formulation of a PTH analog, is in Phase I testing.

"There is a strong need for more anabolic agents to treat osteoporosis," and the Karsenty team's findings in that regard are promising and intriguing, said Thomas Leonard, VP and CSO of Merrion Pharmaceuticals plc. "If such an anabolic agent had only weak side effects, that would be advantageous."

Leonard suggested testing the TPH1 inhibitor in nonrodent models of osteoporosis-such as dogs or monkeys-because murine models do not metabolize bone the same way humans do.

Merrion Pharmaceuticals has completed Phase II testing of Almerol alendronate (MER-103) to treat osteoporosis and is seeking partners for Phase III testing. Almerol is an oral formulation that uses the company's gastrointestinal permeation enhancement technology (GIPET) to improve a compound's bioavailability and reduce its GI side effects. Merck \& Co. Inc. markets Fosamax alendronate to treat osteoporosis.

\section{Stomaching new therapies}

Brian Zambrowicz, EVP and CSO of Lexicon, told SciBX that the company developed LP533401 as a research tool and has not developed it further. Instead, Lexicon and partner Symphony Icon Inc. have two other TPH1 inhibitors in Phase II testing: LX1031 to treat 
irritable bowel syndrome (IBS) and LX1032 to treat GI symptoms associated with carcinoid syndrome.

LX1032 has Fast Track designation in the U.S. and Orphan Drug designation in the EU.

Zambrowicz said the company is conducting studies to evaluate the possibility of developing its TPH1 inhibitors to treat osteoporosis. He declined to disclose details.

MacDonald suggested that Lexicon's TPH1 inhibitors might have to be reoptimized to treat osteoporosis. For example, he said, the high doses of TPH1 inhibitors required for a treatment effect-grams/day of LX1031 in Lexicon's IBS trials and $\mathrm{mg} / \mathrm{kg}$ doses of LP533401 in the Karsenty team's mouse studies - might not be tolerated by osteoporosis patients.

"A high dosing regimen might be OK for IBS because the patients would be motivated to take the drug" by the nature of their symptoms and the drug's ability to relieve them, he said. But osteoporosis patients might not comply with such a dosing regimen because their symptoms and the effects of treatment are less obvious, he said.

Lexicon's Zambrowicz countered that the high doses of LX1031 reflected an important feature of the compound's safety profile-low bioavailability that confined its action primarily to the GI tract. By keeping its effects local, the compound "has been shown to be very safe and have a clinical benefit" in IBS patients, he said.

He added that Lexicon has a more potent TPH1 inhibitor, LX1033, in preclinical development to treat IBS. "LX1033 is 10 times more potent and so would certainly require lower doses" than LX1031, he said.

"Serotonin produced in the peripheral nervous system has a long list of functions" that could be adversely affected by a systemic inhibitor, Zambrowicz noted. "An inhibitor of gut-derived serotonin with systemic exposure would hit serotonin produced elsewhere in the periphery-including the serotonin produced in peripheral nerves that regulate GI motility." Thus, he said confining serotonin inhibitors to the GI tract should produce a wider safety window versus inhibitors with higher bioavailability and consequently higher systemic exposure.

Karsenty noted that inhibition of serotonin production in the gut had observable effects only on bone formation in his team's studies.

He said the team's ongoing work includes additional studies in animal models of osteoporosis and identifying other molecules that inhibit production of gut-derived serotonin.

The findings reported in Nature Medicine are patented by Columbia University, but Karsenty declined to disclose the licensing status of those findings.

Haas, M.J. SciBX 3(7); doi:10.1038/scibx.2010.203

Published online Feb. 18, 2010

\section{REFERENCES}

1. Yadav, V. et al. Nat. Med.; published online Feb. 7, 2010; doi:10.1038/nm.2098

Contact: Gerard Karsenty, Columbia University Medical Center, New York, N.Y. e-mail: gk2172@columbia.edu

2. Liu, Q. et al. J. Pharmacol. Exp. Ther. 325, 47-55 (2008)

3. Yadav, V. et al. Cell 135, 825-837 (2008)

\section{COMPANIES AND INSTITUTIONS MENTIONED}

Central Drug Research Institute, Lucknow, India Columbia University, New York, N.Y.

Columbia University Medical Center, New York, N.Y.

Dana-Farber Cancer Institute, Boston, Mass.

Eli Lilly and Co. (NYSE:LLY), Indianapolis, Ind.

Harvard Medical School, Boston, Mass.

Indian Institute of Science, Bangalore, India

Lexicon Pharmaceuticals Inc. (NASDAQ:LXRX), The Woodlands, Texas

Merck \& Co. Inc. (NYSE:MRK), Whitehouse Station, N.J.

Merrion Pharmaceuticals plc (ISE:MERR), Dublin, Ireland

NPS Pharmaceuticals Inc. (NASDAQ:NPSP), Bedminster, N.J.

Nycomed, Zurich, Switzerland

Symphony Icon Inc., Rockville, Md.

Zelos Therapeutics Inc., West Conshohocken, Pa. 